\title{
Growing drift-Alfvén modes in collisional solar plasma
}

\author{
J. Vranjes and S. Poedts
}

\author{
Center for Plasma Astrophysics, K.U. Leuven, Celestijnenlaan 200B, 3001 Leuven, Belgium \\ e-mail: Jovo.Vranjes@wis.kuleuven.be
}

Received 2 June 2006 / Accepted 28 July 2006

ABSTRACT

\begin{abstract}
Context. Solar plasmas are structured and stratified both vertically and horizontally. The presence of density gradients and magnetic fields results in an additional wave which can be electrostatic (the drift wave) and electromagnetic (the drift-Alfvén wave).

Aims. The stability is discussed of the drift-Alfvén wave which is driven by the equilibrium density gradient, in both unbounded and bounded, collisional solar plasmas, including the effects of both hot ions and a finite ion Larmor radius. The density gradient in combination with the electron collisions with heavier plasma species is the essential source of the instability of the electrostatic drift mode which is coupled to the dispersive Alfvén mode.

Methods. An analytical linear normal mode analysis is used for the description of the waves in spatially unlimited plasma. In the application to the magnetic structures the complex eigen-modes and the corresponding complex discrete eigen-frequencies in cylindric, radially inhomogeneous, collisional and bounded plasma are derived and discussed.

Results. A detailed derivation of the hot ion (the finite ion Larmor radius) contribution is performed within the two fluid model. In the analysis of modes in an unbounded plasma the exchange of identity between the electrostatic and electromagnetic modes is demonstrated. Due to this, the frequency of the electromagnetic part of the mode becomes very different compared to the case without the density gradient. In the case of a bounded plasma the dispersion properties of modes involve a discrete poloidal mode number, and eigen-functions in terms of Bessel functions with discrete zeros at the boundary. The results are applied to coronal and chromospheric plasmas.
\end{abstract}

Key words. waves - instabilities - Sun: corona - Sun: chromosphere

\section{Introduction}

The electrostatic drift wave possesses the unique feature of being unstable both in the fluid domain of a resistive plasma and in the collision-less kinetic description. In both cases the electrons play an essential role; in the former case, the instability is due to their collisions with heavier species, while in the latter case the instability arises due to the Cherenkov type electron interaction with the parallel wave-phase velocity.

For plasmas with a not so small plasma $\beta\left(>m_{\mathrm{e}} / m_{\mathrm{i}}\right)$, the mode becomes electromagnetic and in such plasmas an interplay between the electrostatic and electromagnetic part of the mode takes place. The electromagnetic component is an Alfvéntype perturbation which is dispersive like the kinetic-Alfvén wave (KAW) or the inertial Alfvén wave (IAW). Which of these modes enter the perturbations depends essentially on the plasma $\beta$, yet it also implies a certain range of the wave numbers $k_{\perp}$ in the direction perpendicular to the magnetic field lines. For the $\mathrm{KAW}$, the determining parameter is $k_{\perp} \rho_{s}$, where $\rho_{s}=c_{s} / \Omega_{\mathrm{i}}$, with $c_{s}=\left(\kappa T_{\mathrm{e}} / m_{\mathrm{i}}\right)^{1 / 2}$, and $\Omega_{\mathrm{i}}=e B_{0} / m_{\mathrm{i}}$. For the solar coronal plasma, $\rho_{s}$ is typically of the order of $1 \mathrm{~m}$. For the IAW, the relevant parameter is $k_{\perp} \lambda_{\mathrm{e}}$, where $\lambda_{\mathrm{e}}=c / \omega_{\mathrm{pe}}$, and $\omega_{\mathrm{pe}}=\left(e^{2} n_{0} / \varepsilon_{0} m_{\mathrm{e}}\right)^{1 / 2}$, implying even shorter scales, viz. $<0.5 \mathrm{~m}$. On the other hand, to have modes with reasonably low and detectable frequencies $\sim k_{z} c_{a}$, where $c_{a}=B_{0} /\left(\mu_{0} n_{0} m_{\mathrm{i}}\right)^{1 / 2}$, the parallel wave-length is measured in hundreds of kilometers. The huge difference in the perpendicular and parallel scales is, in fact, favorable for the drift wave instability.

In the present study, we apply the basic theory of the EM drift wave to the solar plasma. This implies high and equal electron and ion temperatures, and possibly short scales in the perpendicular direction. Therefore, we derive and include the ion gyro-viscosity stress tensor terms which, for short wave lengths, appear of the same order as the standard polarization drift terms. They both describe the finite ion Larmor radius (FLR) effects, yet the latter appears due to the time dependence of the diamagnetic drift. Some important features, like the exchange of identity of modes and the related frequency change, will be pointed out.

\section{Model and derivations}

We assume a magnetic field in the $z$-direction, $B_{0} \boldsymbol{e}_{z}$, and an equilibrium plasma density that has a gradient in the perpendicular direction. We then study low-frequency perturbations satisfying the condition $\partial / \partial t \ll \Omega_{\mathrm{i}}$.

The momentum equations for ions and electrons are

$$
m_{\mathrm{i}} n_{\mathrm{i}}\left[\frac{\partial \boldsymbol{v}_{\mathrm{i}}}{\partial t}+\left(\boldsymbol{v}_{\mathrm{i}} \cdot \nabla\right) \boldsymbol{v}_{\mathrm{i}}\right]=e n_{\mathrm{i}}\left(-\nabla \phi-\frac{\partial A_{z}}{\partial t} \boldsymbol{e}_{z}+\boldsymbol{v}_{\mathrm{i}} \times \boldsymbol{B}\right)
$$$$
-\kappa T_{\mathrm{i}} \nabla n_{\mathrm{i}}-\nabla \cdot \Pi_{\mathrm{i}}-m_{\mathrm{i}} n_{\mathrm{i}} v_{\mathrm{i}} v_{\mathrm{i}}
$$

$$
m_{\mathrm{e}} n_{\mathrm{e}}\left[\frac{\partial \boldsymbol{v}_{\mathrm{e}}}{\partial t}+\left(\boldsymbol{v}_{\mathrm{e}} \cdot \nabla\right) \boldsymbol{v}_{\mathrm{e}}\right]=-e n_{\mathrm{i}}\left(-\nabla \phi-\frac{\partial A_{z}}{\partial t} \boldsymbol{e}_{z}+\boldsymbol{v}_{\mathrm{e}} \times \boldsymbol{B}\right)
$$

$-\kappa T_{\mathrm{e}} \nabla n_{\mathrm{e}}-\nabla \cdot \Pi_{\mathrm{e}}-m_{\mathrm{e}} n_{\mathrm{e}}\left(v_{\mathrm{e}} \boldsymbol{v}_{\mathrm{e}}-v_{\mathrm{ei}} \boldsymbol{v}_{\mathrm{i}}\right)$.

Here, $v_{\mathrm{i}} \equiv v_{\text {in }}$ and $v_{\mathrm{e}}=v_{\mathrm{en}}+v_{\mathrm{ei}}$, i.e., we allow for the presence of neutrals in the plasma and, in view of the huge difference in mass, we neglect the ion momentum change due to their collisions with electrons. The model is consequently applicable to 
partially ionized as well as to fully ionized plasmas. In order to apply the model to hot plasmas we retain the stress tensor contribution. More precisely, we keep its leading order gyro-viscous part. In practice, provided we deal with relatively short wavelengths, this gives corrections mainly to the ion dynamics.

In the case of highly magnetized electrons and in the limit when their inertia effects are negligible, the total perpendicular electron velocity in the linear limit reduces to

$\boldsymbol{v}_{\mathrm{e} \perp}=\frac{1}{B_{0}} \boldsymbol{e}_{z} \times \nabla_{\perp} \phi-\frac{\kappa T_{\mathrm{e}}}{e B_{0}} \boldsymbol{e}_{z} \times \frac{\nabla_{\perp} n_{\mathrm{e}}}{n_{\mathrm{e}}}$.

The parallel electron dynamics, in the limit when ion motion is predominantly polarized in the perpendicular plain, is described by

$\left(\frac{\partial}{\partial t}+\boldsymbol{v}_{\mathrm{e} 0} \nabla_{\perp}\right) A_{z 1}+\frac{\partial \phi_{1}}{\partial z}-\frac{\kappa T_{\mathrm{e}}}{n_{\mathrm{e} 0} e} \frac{\partial n_{\mathrm{e} 1}}{\partial z}-\frac{m_{\mathrm{e}} v_{\mathrm{e}}}{\mu_{0} e^{2} n_{\mathrm{e} 0}} \nabla_{\perp}^{2} A_{z 1}=0$

Here, $v_{\mathrm{e} 0}$ denotes the equilibrium electron diamagnetic drift velocity described by the second term in Eq. (3), i.e., $\boldsymbol{v}_{\mathrm{e} 0}=$ $-\kappa T_{\mathrm{e}} \boldsymbol{e}_{z} \times \nabla_{\perp} n_{\mathrm{e} 0} /\left(e B_{0} n_{\mathrm{e} 0}\right)$, and we have also used Ampère law, $\nabla \times \boldsymbol{B}=\mu_{0} \boldsymbol{j}$ yielding $e n_{\mathrm{e} 0} \mu_{0} v_{\mathrm{e} z 1}=\nabla_{\perp}^{2} A_{z 1}$.

The electron continuity becomes

$\frac{\partial n_{\mathrm{e} 1}}{\partial t}+\frac{1}{B_{0}}\left(\boldsymbol{e}_{z} \times \nabla_{\perp} \phi_{1}\right) \cdot \nabla_{\perp} n_{\mathrm{e} 0}+\frac{1}{\mu_{0} e} \frac{\partial}{\partial z} \nabla_{\perp}^{2} A_{z 1}=0$.

The ion perpendicular motion, obtained from Eq. (1), is described by the recurrent formula

$v_{\mathrm{i} \perp}=\alpha_{\mathrm{i}}\left[\frac{1}{B_{0}} \boldsymbol{e}_{z} \times \nabla_{\perp} \phi+\frac{v_{T \mathrm{i}}^{2}}{\Omega_{\mathrm{i}}} \boldsymbol{e}_{z} \times \frac{\nabla_{\perp} n_{\mathrm{i}}}{n_{\mathrm{i}}}-\frac{v_{\mathrm{i}}}{\Omega_{\mathrm{i}}} \frac{\nabla_{\perp} \phi}{B_{0}}\right.$

$-\frac{v_{\mathrm{i}} v_{T \mathrm{i}}^{2}}{\Omega_{\mathrm{i}}^{2}} \frac{\nabla_{\perp} n_{\mathrm{i}}}{n_{\mathrm{i}}}+\boldsymbol{e}_{z} \times \frac{\nabla_{\perp} \cdot \Pi_{\mathrm{i}}}{m_{\mathrm{i}} n_{\mathrm{i}} \Omega_{\mathrm{i}}}+\frac{1}{\Omega_{\mathrm{i}}}\left(\frac{\partial}{\partial t}+\boldsymbol{v}_{\mathrm{i} \perp} \cdot \nabla_{\perp}\right) \boldsymbol{e}_{z} \times \boldsymbol{v}_{\mathrm{i} \perp}$

$\left.-\frac{v_{\mathrm{i}}}{\Omega_{\mathrm{i}}} \frac{\nabla_{\perp} \cdot \Pi_{\mathrm{i}}}{e n_{\mathrm{i}} B_{0}}-\frac{1}{\Omega_{\mathrm{i}}} \frac{v_{\mathrm{i}}}{\Omega_{\mathrm{i}}}\left(\frac{\partial}{\partial t}+\boldsymbol{v}_{\mathrm{i} \perp} \cdot \nabla_{\perp}\right) \boldsymbol{v}_{\mathrm{i} \perp}\right]$.

Here $\alpha_{\mathrm{i}}=1 /\left(1+v_{\mathrm{i}}^{2} / \Omega_{\mathrm{i}}^{2}\right)$. In the case $v_{\mathrm{i}}^{2} \ll \Omega_{\mathrm{i}}^{2}$, we may set $\alpha_{\mathrm{i}} \rightarrow 1$, the first two terms are the leading order ones and should be used in the polarization drift, while the last two terms are higher order terms and can be omitted.

Equation (6) is used in the ion continuity equation to calculate the terms $\nabla_{\perp}\left(n_{\mathrm{i}} v_{\perp \perp}\right)$. The procedure is straightforward except for the term with the convective derivative in the polarization drift $\boldsymbol{v}_{\mathrm{p}}$, i.e., $\left(\boldsymbol{v}_{\mathrm{i} \perp} \cdot \nabla_{\perp}\right) \boldsymbol{e}_{z} \times \boldsymbol{v}_{\mathrm{i} \perp}$, and the stress tensor contribution $\boldsymbol{v}_{\pi}$. For a small equilibrium density gradient, the last $\boldsymbol{v}_{\mathrm{i} \perp}$ in $\boldsymbol{v}_{\mathrm{p}}$ from (6) comprises only the leading order perturbed $\boldsymbol{E} \times \boldsymbol{B}$ and diamagnetic drifts $\left(\boldsymbol{v}_{\mathrm{E} 1}\right.$ and $\left.\boldsymbol{v}_{* 11}\right)$, while the first $\boldsymbol{v}_{\mathrm{i}}$ is the equilibrium ion diamagnetic drift $\boldsymbol{v}_{\mathrm{i} 0}=\kappa T_{\mathrm{i}} \boldsymbol{e}_{z} \times \nabla_{\perp} n_{\mathrm{i} 0} /\left(e B_{0} n_{\mathrm{i} 0}\right)=$ $-v_{\mathrm{e} 0} T_{\mathrm{i}} / T_{\mathrm{e}}$. On the other hand, the stress tensor part after a few steps yields

$$
\begin{aligned}
\nabla_{\perp} \cdot\left(n \boldsymbol{v}_{\pi}\right) & =-\rho_{\mathrm{i}}^{2} \nabla_{\perp} n_{\mathrm{i} 0} \cdot \nabla_{\perp}^{2} \boldsymbol{v}_{\mathrm{i} \perp}-n_{\mathrm{i} 0} \rho_{\mathrm{i}}^{2} \nabla_{\perp}^{2} \nabla_{\perp} \cdot \boldsymbol{v}_{\mathrm{i} \perp} \\
& =-\rho_{\mathrm{i}}^{2} \nabla_{\perp} n_{\mathrm{i} 0} \cdot \nabla_{\perp}^{2} \boldsymbol{v}_{\mathrm{i} \perp}+\frac{\rho_{\mathrm{i}}^{2} n_{\mathrm{i} 0}}{\Omega_{\mathrm{i}}} \frac{\partial}{\partial t} \nabla_{\perp}^{4}\left(\frac{\phi_{1}}{B_{0}}+\frac{v_{\mathrm{Ti}}^{2}}{\Omega_{\mathrm{i}}} \frac{n_{\mathrm{i} 1}}{n_{\mathrm{i} 0}}\right) .
\end{aligned}
$$

The first term in this expression, within the second order approximation limit, cancels out with the term $\left(\boldsymbol{v}_{\mathrm{i} 0} \cdot \nabla_{\perp}\right) \boldsymbol{e}_{z} \times \boldsymbol{v}_{\mathrm{i} \perp}$ from the above discussed convective derivative in the polarization drift which appears in $\nabla_{\perp} \cdot\left(n_{\mathrm{i}} \boldsymbol{v}_{\mathrm{p}}\right)$. The second term in Eq. (7) is the FLR contribution, obtained by substituting the velocity (6) into the operator.
Another similar FLR term is obtained from the time derivative part of $\nabla_{\perp} \cdot\left(n_{\mathrm{i}} \boldsymbol{v}_{\mathrm{p}}\right)$ reading

$-n_{\mathrm{i} 0} \rho_{\mathrm{i}}^{2} \frac{\partial}{\partial t} \nabla_{\perp}^{2} \frac{e \phi_{1}}{\kappa T_{\mathrm{i}}}-\rho_{\mathrm{i}}^{2} \frac{\partial}{\partial t} \nabla_{\perp}^{2} n_{\mathrm{i} 1}$.

Here, $\rho_{\mathrm{i}}=v_{T \mathrm{i}} / \Omega_{\mathrm{i}}$, and $v_{T \mathrm{i}}^{2}=\kappa T_{\mathrm{i}} / m_{\mathrm{i}}$.

The ion continuity equation finally yields

$$
\begin{aligned}
& \frac{\partial}{\partial t}\left(\frac{n_{\mathrm{i} 1}}{n_{\mathrm{i} 0}}\right)+\frac{1}{B_{0}} \boldsymbol{e}_{z} \times \nabla_{\perp} \phi_{1} \cdot \frac{\nabla_{\perp} n_{\mathrm{i} 0}}{n_{\mathrm{i} 0}}-v_{\mathrm{i}} n_{\mathrm{i} 0} \rho_{\mathrm{i}}^{2} \nabla_{\perp}^{2}\left(\frac{e \phi_{1}}{\kappa T_{\mathrm{i}}}+\frac{n_{\mathrm{i} 1}}{n_{\mathrm{i} 0}}\right) \\
& -n_{\mathrm{i} 0} \rho_{\mathrm{i}}^{2} \frac{\partial}{\partial t} \nabla_{\perp}^{2}\left(\frac{e \phi_{1}}{\kappa T_{\mathrm{i}}}+\frac{n_{\mathrm{i} 1}}{n_{\mathrm{i} 0}}\right)+\frac{\rho_{\mathrm{i}}^{2} n_{\mathrm{i} 0}}{\Omega_{\mathrm{i}}} \frac{\partial}{\partial t} \nabla_{\perp}^{4}\left(\frac{\phi_{1}}{B_{0}}+\frac{v_{T \mathrm{i}}^{2}}{\Omega_{\mathrm{i}}} \frac{n_{\mathrm{i} 1}}{n_{\mathrm{i} 0}}\right)=0 .
\end{aligned}
$$

Equations (5) and (9) are combined using the quasi-neutrality, yielding a more convenient form of the third equation which closes the system, viz.

$$
\begin{aligned}
& \left(\frac{\partial}{\partial t}+v_{\mathrm{i}}\right) \nabla_{\perp}^{2} \phi_{1}+c_{a}^{2} \frac{\partial}{\partial z} \nabla_{\perp}^{2} A_{z 1}+\frac{\kappa T_{\mathrm{i}}}{e n_{0}}\left(\frac{\partial}{\partial t}+v_{\mathrm{i}}\right) \nabla_{\perp}^{2} n_{1} \\
& -\rho_{\mathrm{i}}^{2} \frac{\partial}{\partial t} \nabla_{\perp}^{4}\left(\phi_{1}+\frac{\kappa T_{\mathrm{i}}}{e n_{0}} n_{1}\right)=0 .
\end{aligned}
$$

The given set of Eqs. (4), (5), and (10) will be used in the description of the drift-Alfvén waves in solar plasma.

\section{Waves in unlimited plasma}

In Cartesian geometry, for perturbations $\sim \exp \left(-\mathrm{i} \omega t+\mathrm{i} k_{y} y+\mathrm{i} k_{z} z\right)$, Eqs. (4), (5), and (10) yield

$$
\begin{aligned}
& \omega^{3}-\omega^{2}\left[\omega_{* \mathrm{e}}+\omega_{* \mathrm{i}}-\mathrm{i}\left(\delta+\frac{v_{\mathrm{i}}}{1+k_{y}^{2} \rho_{\mathrm{i}}^{2}}\right)\right] \\
& +\omega\left\{\omega_{* \mathrm{e}} \omega_{* \mathrm{i}}-\frac{k_{z}^{2} c_{a}^{2}}{1+k_{y}^{2} \rho_{\mathrm{i}}^{2}}-k_{y}^{2} k_{z}^{2} c_{a}^{2}\left(\rho_{s}^{2}+\rho_{\mathrm{i}}^{2}\right)-\frac{v_{\mathrm{i}} \delta}{1+k_{y}^{2} \rho_{\mathrm{i}}^{2}}\right. \\
& \left.-\mathrm{i}\left[\omega_{* \mathrm{i}} \delta+\frac{v_{\mathrm{i}}\left(\omega_{* \mathrm{e}}+\omega_{* \mathrm{i}}\right)}{1+k_{y}^{2} \rho_{\mathrm{i}}^{2}}\right]+\frac{\omega_{* \mathrm{e}} k_{z}^{2} c_{a}^{2}}{1+k_{y}^{2} \rho_{\mathrm{i}}^{2}}+\frac{\omega_{* \mathrm{i}} v_{\mathrm{i}} \delta}{1+k_{y}^{2} \rho_{\mathrm{i}}^{2}}\right\} \\
& +\mathrm{i} \frac{v_{\mathrm{i}}}{1+k_{y}^{2} \rho_{\mathrm{i}}^{2}}\left[\omega_{* \mathrm{e}} \omega_{* \mathrm{i}}-k_{z}^{2} c_{a}^{2} k_{y}^{2}\left(\rho_{s}^{2}+\rho_{\mathrm{i}}^{2}\right)\right]=0 .
\end{aligned}
$$

Here, $\omega_{* \mathrm{e}}=k_{y} v_{\mathrm{e} 0}, v_{\mathrm{e} 0}=-\kappa T_{\mathrm{e}} \kappa_{0} /\left(e B_{0}\right), \kappa_{0}=n_{0}^{\prime} / n_{0}, \omega_{* \mathrm{i}}=k_{y} v_{\mathrm{i} 0}$, $v_{\mathrm{i} 0}=\kappa T_{\mathrm{i}} \kappa_{0} /\left(e B_{0}\right), \delta=m_{\mathrm{e}} v_{\mathrm{e}} k_{y}^{2} /\left(\mu_{0} n_{0} e^{2}\right)$.

In the collision-less limit and for $T_{\mathrm{e}}>T_{\mathrm{i}}$, and after the expansion $\left(1+k_{y}^{2} \rho_{\mathrm{i}}^{2}\right)^{-1} \simeq 1-k_{y}^{2} \rho_{\mathrm{i}}^{2}$, Eq. (11) yields the drift-Alfvén mode derived earlier by using the kinetic theory (Weiland 2000):

$\omega^{3}-\omega^{2}\left(\omega_{* \mathrm{e}}+\omega_{* \mathrm{i}}\right)-\omega\left[k_{z}^{2} c_{a}^{2}-\omega_{* \mathrm{e}} \omega_{* \mathrm{i}}+k_{z}^{2} c_{a}^{2} k_{y}^{2} \rho_{s}^{2}\right]$

$+k_{z}^{2} c_{a}^{2}\left(\omega_{* \mathrm{e}}+k_{y}^{2} \rho_{s}^{2} \omega_{* \mathrm{i}}\right)=0$.

In the limit of small $k_{y} \rho_{s}$ it reduces to

$\left(\omega-\omega_{* \mathrm{e}}\right)\left[\omega^{2}-\omega \omega_{* \mathrm{i}}-k_{z}^{2} c_{a}^{2}\right]=0$,

yielding three obvious solutions, i.e., the electrostatic drift wave and two (accelerated and retarded) Alfvén waves (Krall \& Trivelpiece 1973):

$\omega_{1}=\omega_{* \mathrm{e}}, \quad \omega_{2,3}=\frac{1}{2}\left[\omega_{* \mathrm{i}} \pm \omega_{* \mathrm{i}}\left(1+\frac{4 k_{z}^{2} c_{a}^{2}}{\omega_{* \mathrm{i}}^{2}}\right)^{1 / 2}\right]$. 
For small $k_{z}$ the two latter waves become

$\omega_{2} \simeq \omega_{* \mathrm{i}} \equiv-\frac{\omega_{* \mathrm{e}} T_{\mathrm{i}}}{T_{\mathrm{e}}}, \quad \omega_{3}=\frac{k_{z}^{2} c_{a}^{2}}{\left|\omega_{* \mathrm{i}}\right|}$.

Hence, the actual frequencies of the modes in a hot plasma with density gradients in the direction perpendicular to the magnetic field lines, may become very different from the frequencies of the standard Alfvén modes propagating in opposite directions, i.e. $\pm k_{z} c_{a}$. This fact should be taken into account in fitting observations into the modeling of solar coronal plasmas.

In the limit of negligible ion thermal effects Eq. (11) reduces to

$\omega^{3}-\omega^{2} \omega_{*}-\omega k_{z}^{2} c_{a}^{2}\left(1+k_{y}^{2} \rho_{s}^{2}\right)+\omega_{*} k_{z}^{2} c_{a}^{2}+\mathrm{i} v_{\mathrm{e}} k_{y}^{2} \lambda_{\mathrm{e}}^{2} \omega^{2}=0$

In the absence of a density gradient this yields a damped kineticAlfvén mode (Vranjes et al. 2006). On the other hand, in the limit $\omega^{2} \ll k_{z}^{2} c_{a}^{2}$ we have a standard unstable drift mode (Vranjes \& Poedts 2006a) with frequency $\omega_{r}=\omega_{* \mathrm{e}} /\left(1+k_{y}^{2} \rho_{s}^{2}\right)$ and increment $\omega_{\mathrm{i}}=v_{\mathrm{e}} \omega_{* \mathrm{e}}^{2} k_{y}^{2} \rho_{s}^{2} /\left(k_{z}^{2} v_{T \mathrm{e}}^{2}\right)$. This is a unique feature of the drift mode, which is intrinsically unstable in a collisional plasma. The instability appears as a common effect of the electron collisions $v_{\mathrm{e}}$, the finite ion mass effect (the term $k_{y}^{2} \rho_{s}^{2}$ ), and the equilibrium density gradient (the term $\omega_{* \mathrm{e}}$ ). The two modes are coupled even without collisions, when we have $\left(\omega-\omega_{* \mathrm{e}}\right)\left(\omega^{2}-k_{z}^{2} c_{a}^{2}\right)=\omega k_{z}^{2} c_{a}^{2} k_{y}^{2} \rho_{s}^{2}$, and the coupling vanishes in the limit of negligible $k_{y}^{2} \rho_{s}^{2}$, i.e., for the case of the drift and the non-dispersive Alfvên modes. Clearly, in order to have an electromagnetic drift-Alfvén mode distinguishable from the ion sound mode, the scales along the magnetic field should be much larger compared to those in the perpendicular direction. Hence, to have a reasonable coupling between the drift and kinetic-Alfvén part, and negligible parallel ion dynamics, $k_{y} \rho_{s}$ should not be too small, and the parallel wave-length must satisfy (Goldston \& Rutherford 1995)

$\lambda_{z}>\frac{2 \pi}{k_{y} \rho_{s}} L_{n}$,

otherwise, we would in principle have a threshold for the instability of the drift mode, viz. $\omega_{r}>k_{z} c_{s}$. Here, $L_{n}$ is the characteristic scale length for the inhomogeneous density. The condition (15) can be satisfied even under laboratory conditions and especially in the present solar plasma case with very large vertical scales. In the same time, the omitted electron inertia effects imply $v_{T \mathrm{e}}>c_{a}$, which is equivalent to the plasma $\beta>m_{\mathrm{e}} / m_{\mathrm{i}}$ and to the limit which requires the inclusion of electromagnetic effects (Weiland 2000).

Although Eqs. (11) and (14) are relatively simple and cubic only, calculating and discussing exact solutions of these equations appears practically rather inconvenient. This is seen in the case of Eq. (14) which can be easily analytically solved, yielding three complex solutions which, however, are nontrivial to discuss. Yet, to some extent the character of the solutions may be understood even without directly solving the dispersion equations, i.e., by using the generalized Hurwitz method for polynomials with complex coefficients (Giaretta 1979). For a general polynomial of the degree $m$ and with complex coefficients, $x^{m}+\left(a_{1}+\mathrm{i} b_{1}\right) x^{m-1}+\ldots+\left(a_{m}+\mathrm{i} b_{m}\right)=0$, one constructs the

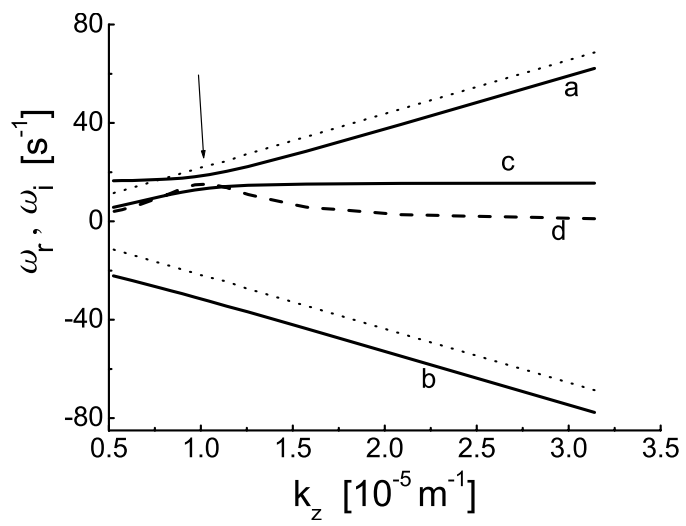

Fig. 1. Frequencies $\omega_{r}$ and increment $\omega_{\mathrm{i}}$ of electromagnetic the driftAlfvén perturbations with the effect of the coupling between the Alfvén (lines a and b) and drift (line c) parts. Dotted lines denote $\pm k_{z} c_{a}$. The increment of the electrostatic drift mode (multiplied by $10^{3}$ ) has a maximum in the region where the retarded kinetic-Alfvén mode and the drift mode change their identities (denoted by arrow).

sequence of $m+1$ numbers $c_{0}=1, c_{1}=a_{1}, \ldots, c_{r}, \ldots$, where $r$ goes to $m$, and where

$c_{r}=(-1)^{r(r-1) / 2}\left|\begin{array}{cccccc}a_{1} & 1 & 0 & 0 & 0 & \cdot \\ -b_{2} & -b 1 & a_{1} & 1 & 0 & \cdot \\ a_{3} & a_{2} & b_{2} & b_{1} & a_{1} & \cdot \\ -b_{4} & -b_{3} & a_{3} & a_{2} & -b_{2} & \cdot \\ a_{5} & a_{4} & b_{4} & b_{3} & a_{3} & \cdot \\ \cdot & \cdot & \cdot & \cdot & \cdot & \cdot \\ \cdot & \cdot & \cdot & \cdot & \cdot & \cdot \\ a_{2 r-1} & a_{2 r-2} & b_{2 r-2} & b_{2 r-3} & a_{2 r-3}\end{array}\right|$.

According to Giaretta (1979), the number of roots with positive real parts equals the number of sign changes in the sequence $c_{j}$. A sufficient instability condition is that any of the $c_{r}$ has a negative sign. As a simple check, we apply the method on Eq. (14). Here, we find $c_{0}=1, c_{1}=-\omega_{*}, c_{2}=-\omega_{*}^{2} k_{z}^{2} k_{y}^{2} c_{a}^{2} \rho_{s}^{2}<0$, $c_{3}=c_{a}^{4} k_{z}^{4} \omega_{*}^{3}\left(\delta^{2}+c_{a}^{2} k_{y}^{4} k_{z}^{2} \rho_{s}^{4}\right)>0$. Consequently, we have two sign changes in the sequence $c_{j}$, i.e., two positive real roots, and $c_{1}$ and $c_{2}$ are both negative, therefore, there exists at least one unstable mode.

\subsection{Coronal plasma}

To discuss the roots and the increments/decrements in detail, we solve Eq. (11) numerically by taking parameters values that are typical for the solar atmosphere. Setting values that may be assumed as typical for the quiet inner solar corona, viz. $T_{\mathrm{e}}=T_{\mathrm{i}}=1.5 \times 10^{6} \mathrm{~K}, n_{\mathrm{e} 0}=n_{\mathrm{i} 0}=10^{14} \mathrm{~m}^{-3}$, and taking $B_{0}=10^{-3} \mathrm{~T}$, we calculate the spectrum for the two oppositely propagating Alfvén modes and the drift mode. Here, neutrals are absent and the dominant collisions take place between electrons and ions, $v_{\mathrm{ei}}=\left(8 \pi / m_{\mathrm{e}}\right)^{1 / 2}\left[e^{2} /\left(4 \pi \varepsilon_{0}\right)\right]^{2} n_{\mathrm{e} 0} L_{\mathrm{ei}} /\left(\kappa T_{\mathrm{e}}\right)^{3 / 2}$, $L_{\mathrm{ei}}=\log \left[12 \pi \varepsilon_{0}\left(\varepsilon_{0} / n_{\mathrm{e} 0}\right)^{1 / 2}\left(\kappa T_{\mathrm{e}}\right)^{3 / 2} / e^{3}\right]$. For these parameters we obtain $v_{\mathrm{ei}} \simeq 2.1 \mathrm{~Hz}, v_{\mathrm{ii}} \simeq 0.07 \mathrm{~Hz}, c_{s}=1.11 \times 10^{5} \mathrm{~m} / \mathrm{s}$, $c_{a}=2.18 \times 10^{6} \mathrm{~m} / \mathrm{s}, v_{T \mathrm{e}}=4.77 \times 10^{6} \mathrm{~m} / \mathrm{s}, \beta=0.0052>$ $m_{\mathrm{e}} / m_{\mathrm{i}}=0.00054$.

The behavior of the modes in terms of the parallel wavenumber $k_{z}$ is presented in Fig. 1 for $L_{n}=10^{3} \mathrm{~m}$ and $\lambda_{y}=50 \mathrm{~m}$, while $k_{y} \rho_{s}=0.15$. We thus have a situation similar to that described by Eq. (12), i.e., two (retarded and accelerated) damped kinetic-Alfvén modes, lines a and b, respectively, and an electrostatic drift mode (line c). The increment of the drift mode 


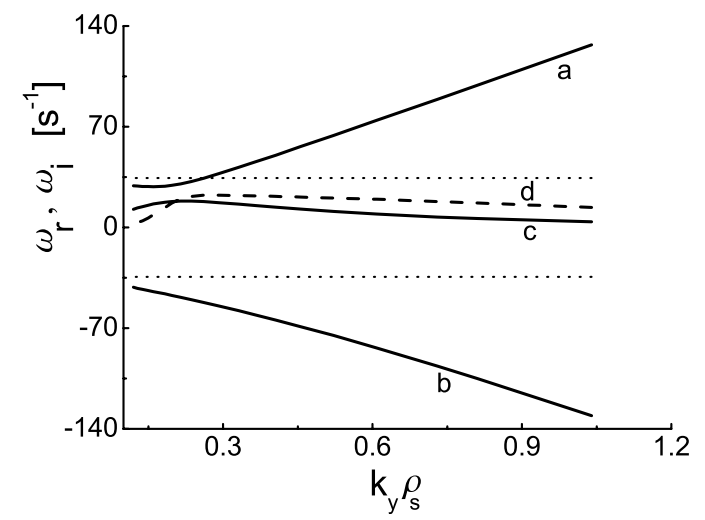

Fig. 2. Frequencies $\omega_{r}$ and increment $\omega_{\mathrm{i}}$ of the drift-Alfvén perturbations in terms of the coupling term $k_{y} \rho_{s}$. The drift wave increment (line d) is multiplied by $10^{3}$. Dotted lines denote $\left|k_{z} c_{a}\right|=34.3 \mathrm{~Hz}$.

(multiplied by $10^{3}$ ) is presented by line $\mathrm{d}$. The drift mode is unstable in the whole range of wave-numbers. Its frequency is nearly constant for large values of $k_{z}$. In the area denoted by the arrow, the retarded kinetic-Alfvén mode and the drift mode do not cross each other. Instead, they change identities as typical for an "avoided crossing". For observations, the low frequency (small $k_{z}$ ) domain is of particular importance as this parameter can be measured, i.e. spatially resolved. It is clearly seen in Fig. 1 that, in fact, this is the domain in which the Alfvén mode frequency can be very different from what is expected or predicted if the small-scale plasma inhomogeneity, which drives the drift mode, is neglected. Here, for the given density scale length, the two frequency limits at $k_{z} \rightarrow 0$ are $\pm 16.26 \mathrm{~Hz}$. Clearly, the frequencies can be made arbitrary small by changing the equilibrium density scale length $L_{N}$.

The decrement of the Alfvén modes has also been calculated and, in general, the accelerated mode $b$ is less damped. Its damping rate changes between $-3.5 \times 10^{-3} \mathrm{~Hz}$ at $k_{z}=3.14$ (in given units), and $-1.1 \times 10^{-3} \mathrm{~Hz}$ at $k_{z}=0.5$. The damping rate of the decelerated mode $a$ has a maximum absolute value of about $2.3 \times 10^{-2} \mathrm{~Hz}$.

In Fig. 2 we present the mode behavior in terms of the coupling term $k_{y} \rho_{s}$ for a fixed value of $\left|k_{z} c_{a}\right|=34.3 \mathrm{~Hz}$ (presented by dotted lines). The notation corresponds to the one used in Fig. 1. Note that at $k_{y} \rho_{s}=1$ the frequencies of the two kineticAlfvén modes are around 127 and $-131 \mathrm{~Hz}$. Thus, the actual frequencies may drastically differ from what is expected without the drift mode.

The drift mode frequency is normally proportional to $1 / L_{N}$. However, due to the coupling with the Alfvén modes, its behavior is also drastically changed. This is seen in Fig. 3 where we fix $k_{y} \rho_{s}=0.52$ and $k_{z}=400 \mathrm{~km}$. Here, contrary to what may be expected, for small $L_{N}$ the mode vanishes and the decrement decreases. This is again due to the identity change with the Alfvén mode. As a matter of fact, the Alfvén frequency for the given numbers is constant $k_{z} c_{a}=34.3 \mathrm{~Hz}$ and the KAWs frequencies (see Fig. 4) at large $L_{N}$ do not change much. For a decreasing $L_{N}$ the drift mode curve does not intersect with the Alfvén mode. Instead, the Alfvén mode takes over the behavior of the drift mode: it grows while the drift mode decreases.

Note that, for the given parameters, the drift curve changes its direction at frequencies around $12 \mathrm{~Hz}$. This is still far enough from the requirement of a small parallel wave-phase velocity in comparison with the electron thermal velocity used in order to omit the electron inertia. Here, we have $k_{z} v_{T e}=75 \mathrm{~Hz}$. Thus, the

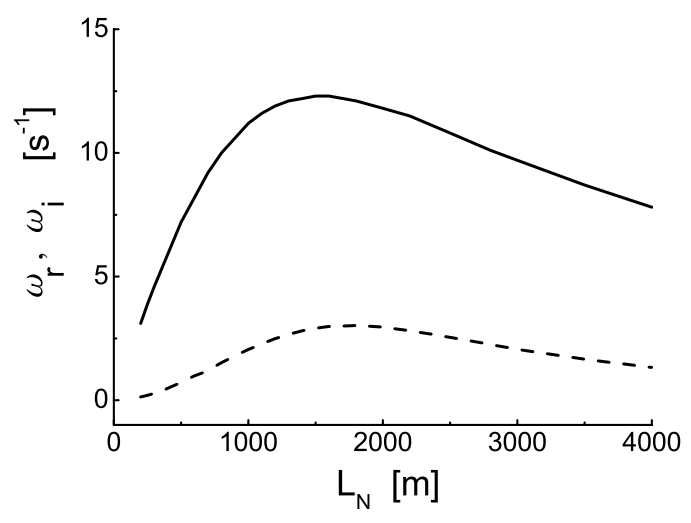

Fig. 3. The drift wave frequency (full line), and its increment multiplied by 100 (dashed line) in terms of the density scale-length, corresponding to the Alfvén modes from Fig. 4.

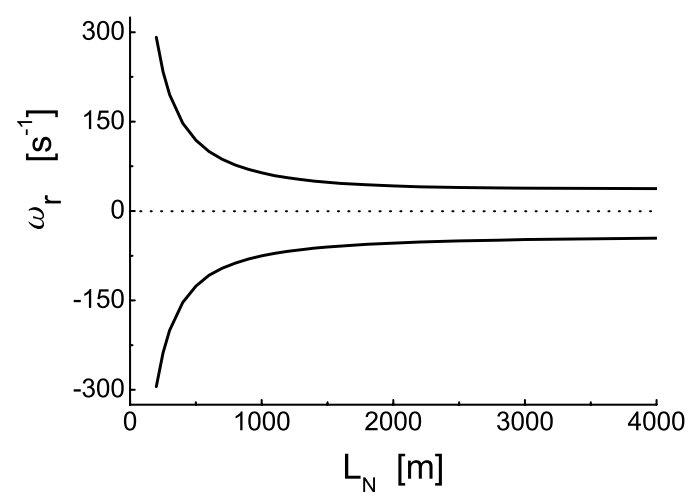

Fig. 4. The frequency of the kinetic-Alfvén modes corresponding to the drift mode in Fig. 3, for the given coupling parameter $k_{y} \rho_{s}=0.52$ and $k_{z} c_{a}=34.3 \mathrm{~Hz}$, in terms of the density scale length.

inclusion of the electron inertia terms is not expected to considerably change the mode behavior.

We note that a similar sort of identity change of the driftAlfvén mode, known in the literature (Weiland 2000), happens also in the case when the ion parallel dynamics is retained, and on the condition that $c_{a}>c_{s}$. In this case, the sound part of the drift mode is disconnected and the parallel mode dependence goes along the line $k_{z} c_{a}$.

\subsection{Application to the chromosphere}

Equation (11) is solved also for the quiet Sun parameters of the chromospheric plasma (Vernazza et al. 1981). Here, starting from the altitude of about $2100-2200 \mathrm{~km}$ and below, the neutral atoms concentration is higher compared to the ions concentration. Within the same range of the parallel wave lengths as in the previous text, the frequencies of the Alfvén modes and the drift mode become below $1 \mathrm{~Hz}$, thus well within the limits of detection. Yet, the ion-neutral collisions appear strong enough to damp all three modes in the given wave length range. However, in this region the parameters change rapidly with the altitude so that it has no sense to consider such long parallel wave lengths. Setting $T=3.2 \times 10^{4} \mathrm{~K}$, and $n_{n 0}=n_{\mathrm{i} 0}=1.5 \times 10^{16} \mathrm{~m}^{-3}$, it turns out that for a short density scale $L_{N} \simeq 10^{2} \mathrm{~m}$, and for $\lambda_{y} \simeq 10 \mathrm{~m}$ and $\lambda_{z} \simeq 10 \mathrm{~km}$, the drift mode is highly unstable, with a frequency $\omega_{r} \simeq 16 \mathrm{~Hz}$ and increment $\omega_{\mathrm{i}} \simeq 2 \mathrm{~Hz}$. Here, $v_{\text {in }} \simeq 2 \mathrm{~Hz}$ and $v_{\mathrm{e}} \simeq 6 \times 10^{4} \mathrm{~Hz}$. 


\section{Eigen-modes in bounded plasma}

In order to present the mode behavior in magnetic structures that are highly elongated along the magnetic field lines and localized in the perpendicular direction, we shall rewrite the starting equations in cylindric coordinates. We use

$\nabla_{r, \theta}=\boldsymbol{e}_{r} \frac{\partial}{\partial r}+\frac{\boldsymbol{e}_{\theta}}{r} \frac{\partial}{\partial \theta}, \quad \nabla_{r, \theta}^{2}=\frac{\partial^{2}}{\partial r^{2}}+\frac{1}{r} \frac{\partial}{\partial r}+\frac{1}{r^{2}} \frac{\partial^{2}}{\partial \theta^{2}}$,

and consider perturbations of the form $\widehat{f}(r) \exp \left(-\mathrm{i} \omega t+\mathrm{i} m \theta+\mathrm{i} k_{z}\right)$, where $\widehat{f}(r)$ denotes the $r$-dependent amplitude and $m$ the discrete poloidal mode number. In the same frame we have $\boldsymbol{v}_{(\mathrm{e}, \mathrm{i}) 0}=$ $\mp \boldsymbol{e}_{\theta} K T_{\mathrm{e}, \mathrm{i}} n_{0}^{\prime} /\left(e B_{0} n_{0}\right)=v_{(\mathrm{e}, \mathrm{i}) 0}(r) \boldsymbol{e}_{\theta}, n_{0}^{\prime}=\mathrm{d} n_{0} / \mathrm{d} r$, with the minus sign for electrons. The combined electron dynamics Eqs. (4) and (5) yield:

$\left(\nabla_{\perp}^{2}+\frac{\frac{\omega \omega_{2}}{c_{a}^{2} k_{z}^{2} \rho_{s}^{2}}}{1-\frac{\mathrm{i} v_{\mathrm{e}} \omega}{k_{z}^{2} v_{T \mathrm{e}}^{2}}}\right) \widehat{A}_{z 1}-\frac{1}{k_{z} c_{a}^{2}} \frac{\frac{\omega}{\rho_{s}^{2}}+\frac{m \Omega_{\mathrm{i}}}{r} \frac{n_{0}^{\prime}}{n_{0}}}{1-\frac{\mathrm{i} v_{\mathrm{e}} \omega}{k_{z}^{2} v_{\mathrm{Te}}^{2}}} \widehat{\phi}_{1}=0$,

where $\omega_{2}=\omega-v_{\mathrm{e} 0}(r) m / r$, and $\nabla_{\perp}=\partial^{2} / \partial r^{2}+\partial /(r \partial r)-m^{2} / r^{2}$. The ion part can be discussed in two limits.

\subsection{Negligible ion thermal effects}

In this limit, from Eq. (10) we obtain

$\nabla_{\perp}^{2} \widehat{\phi}_{1}-\frac{k_{z}^{2} c_{a}^{2}}{\omega} \nabla_{\perp}^{2} \widehat{A}_{z 1}=0$

which is used in Eq. (16) yielding

$\nabla_{\perp}^{2}\left[\left(\nabla_{\perp}^{2}-\frac{1}{\omega\left(1-\mathrm{i} \delta_{1}\right)}\left(\frac{\omega}{\rho_{s}^{2}}+\frac{m \Omega_{\mathrm{i}}}{r} \frac{n_{0}^{\prime}}{n_{0}}\right)\right) \widehat{\phi}_{1}\right]$

$+\nabla_{\perp}^{2}\left[\frac{\omega-m v_{\mathrm{e} 0} / r}{k_{z} \rho_{S}^{2}\left(1-\mathrm{i} \delta_{1}\right)} \widehat{A}_{z 1}\right]=0$

To further decouple the equations for the potentials we have to assume a density profile. We assume a realistic case, viz. $n_{0}(r)=$ $N_{0} \exp \left(a r^{2} / 2\right)$, where $a$ can be both positive and negative, and $r$ takes values between 0 and $r_{0}$. All the terms under the operators in this case become constant and Eq. (18), after using (17) again, becomes of the form

$\nabla_{\perp}^{2}\left\{\left[\nabla_{\perp}^{2}-\frac{\omega / \rho_{s}^{2}+a m \Omega_{\mathrm{i}}}{\omega\left(1-\mathrm{i} \delta_{1}\right)}+\frac{\omega\left(\omega+a m \kappa T_{\mathrm{e}} / e B_{0}\right)}{k_{z}^{2} \rho_{s}^{2} c_{a}^{2}\left(1-\mathrm{i} \delta_{1}\right)}\right] \widehat{\phi}_{1}\right\}=0$.

or

$\nabla_{\perp}^{2} \psi(r)=0$.

Here, consequently

$\left[\nabla_{\perp}^{2}-\frac{\omega / \rho_{s}^{2}+a m \Omega_{\mathrm{i}}}{\omega\left(1-\mathrm{i} \delta_{1}\right)}+\frac{\omega\left(\omega+a m \kappa T_{\mathrm{e}} / e B_{0}\right)}{k_{z}^{2} \rho_{s}^{2} c_{a}^{2}\left(1-\mathrm{i} \delta_{1}\right)}\right] \widehat{\phi}_{1}=\psi(r)$,

and from Eq. (20) $\psi(r)$ is

$\psi(r)=0, \quad$ or $\quad \psi(r)=c_{1} \cosh [m \log (r)]+c_{2} \sinh [m \log (r)]$.

Equation (21) can be readily written in the form

$\left(\frac{\partial^{2}}{\partial r^{2}}+\frac{1}{r} \frac{\partial}{\partial r}-\frac{m^{2}}{r^{2}}+\xi^{2}\right)\left[\widehat{\phi}_{1}-\frac{\psi(r)}{\xi^{2}}\right]=0$, where

$\xi^{2}=\frac{\omega\left(\omega+\omega_{0}\right)}{\rho_{s}^{2}(1-\mathrm{i} \delta)}\left(\frac{1}{c_{a}^{2} k_{z}^{2}}-\frac{1}{\omega^{2}}\right), \quad \omega_{0}=\operatorname{am} \frac{\kappa T_{\mathrm{e}}}{e B_{0}}, \quad \delta_{1}=\frac{v_{\mathrm{e}} \omega}{k_{z}^{2} v_{T \mathrm{e}}^{2}}$.

The solutions of Eq. (23) are the Bessel functions of the first and the second kind, $J_{n}(\xi r)$ and $Y_{n}(\xi r)$, with a complex argument. For nonsingular eigen-functions we keep $J_{n}(\xi r)$. According to the theorems of Lommel \& Bourget (Watson 1962) we have, first, that if $n>-1$, then the zeros of the Bessel function $J_{n}(z)$ with the complex argument $z$ are all real, and, second, for $n \geq 0$ the functions $J_{n}(z)$ and $J_{n+s}(z)$ have no common zeros other than the origin, for all $s>0$. Hence, for vanishing solutions at the boundary, we set that $\xi r_{0}=\varepsilon_{l}$ where $\varepsilon_{l}$ is the real $l$ th zero of the complex function $J_{n}(\xi r)$. This allows us to write the dispersion equation for the radially bounded plasma

$\left(\omega+\operatorname{am} \frac{\kappa T_{\mathrm{e}}}{e B_{0}}\right)\left(\omega^{2}-k_{z}^{2} c_{a}^{2}\right)=\omega k_{z}^{2} c_{a}^{2} \frac{\varepsilon_{l}^{2} \rho_{s}^{2}}{r_{0}^{2}}(1-\mathrm{i} \delta)$.

This is the equivalent of Eq. (14) in an unbounded plasma. Yet, here both $m$ and $\varepsilon_{l}$ take given discrete values. Equation (24) describes the global drift-Alfvén wave, with an unstable drift wave part. The poloidal (i.e. in the $\theta$-direction) propagation is the consequence of the drift mode which propagates perpendicular to both the magnetic field lines and the density gradient. Combined with the given $z$-dependence, this gives the twisting of the global modes (Vranjes \& Poedts 2004a,b). The twisting vanishes for $m=0$ when the two modes decouple. The eigen-functions $\widehat{A}_{z 1}$ can be easily found from Eq. (17), and $\widehat{n}_{1}$ from Eq. (5), which is rewritten as

$\widehat{n}_{1}=\frac{k_{z}}{\mu_{0} e \omega} \nabla_{\perp}^{2} \widehat{A}_{z 1}-\frac{m}{r} \frac{n_{0}^{\prime}}{\omega B_{0}} \widehat{\phi}_{1}$.

Mode details are available in the recent reference Vranjes \& Poedts (2006b).

\subsection{The hot ion case}

Ion thermal effects enter the equations through (8), the second term in (7), and the collisions. Using Eq. (25) in (10) for the same Gaussian/inverse-Gaussian density profile as before, we obtain an equation containing terms proportional to $\rho_{\mathrm{i}}^{4} \nabla_{\perp}^{4} \widehat{\phi}_{1}$ and $\rho_{\mathrm{i}}^{6} \nabla_{\perp}^{6} \widehat{A}_{z 1}$, which come from the second part of the stress tensor in Eq. (7). This 6th-order differential equation is to be combined with the 2nd-order Eq. (16) in order to decouple the two potentials. In the case of the global modes studied here and, therefore, for large scales, we have $\left|\rho_{\mathrm{i}} \nabla_{\perp}\right|<1$ and the high order derivatives yield small terms that can be neglected. Hence, by omitting the stress tensor contribution while still keeping the ion thermal effects through (8) and the collisions, from Eq. (10) we have

$\nabla_{\perp}^{2}\left\{\widehat{\phi}_{1}-\frac{k_{z} c_{a}^{2}}{\left(\omega+\mathrm{i} v_{\mathrm{i}}\right)\left(\omega-m a \rho_{\mathrm{i}}^{2} \Omega_{\mathrm{i}}\right)}\left[\omega-\left(\omega+\mathrm{i} v_{\mathrm{i}}\right) \rho_{\mathrm{i}}^{2} \nabla_{\perp}^{2}\right] \widehat{A}_{z 1}\right\}=0$.

This gives

$\widehat{\phi}_{1}=\frac{k_{z}^{2} c_{a}^{2}}{\left(\omega+\mathrm{i} v_{\mathrm{i}}\right)\left(\omega-m a \rho_{\mathrm{i}}^{2} \Omega_{\mathrm{i}}\right)}\left[\omega-\rho_{\mathrm{i}}^{2}\left(\omega+\mathrm{i} v_{\mathrm{i}}\right) \nabla_{\perp}^{2}\right] \widehat{A}_{z 1}+\psi(r)$,

which is used in Eq. (16) yielding

$\left(\frac{\partial^{2}}{\partial r^{2}}+\frac{1}{r} \frac{\partial}{\partial r}-\frac{m^{2}}{r^{2}}+\eta^{2}\right)\left[\widehat{A}_{z 1}+c \psi(r)\right]=0$ 
Here,

$$
\begin{aligned}
\eta^{2} & =\left[\omega\left(\omega+\mathrm{i} v_{\mathrm{i}}\right)\left(\omega+m a \rho_{s}^{2} \Omega_{\mathrm{i}}\right)\left(\omega-\operatorname{ma\rho }_{\mathrm{i}}^{2} \Omega_{\mathrm{i}}\right)\right. \\
& \left.-\omega k_{z}^{2} c_{a}^{2}\left(\omega+m a \rho_{s}^{2} \Omega_{\mathrm{i}}\right)\right]\left\{k _ { z } ^ { 2 } c _ { a } ^ { 2 } ( \omega + \mathrm { i } v _ { \mathrm { i } } ) \left[\rho_{s}^{2}\left(1-\mathrm{i} \delta_{1}\right)\left(\omega-m a \rho_{\mathrm{i}}^{2} \Omega_{\mathrm{i}}\right)\right.\right. \\
& \left.\left.+\rho_{\mathrm{i}}^{2}\left(\omega+m a \rho_{s}^{2} \Omega_{\mathrm{i}}\right)\right]\right\}^{-1},
\end{aligned}
$$

and

$$
c=\frac{\left(\omega+m a \rho_{s}^{2} \Omega_{\mathrm{i}}\right)}{k_{z} c_{a}^{2} \rho_{s}^{2} \eta^{2}\left(1-\mathrm{i} \delta_{1}\right)} .
$$

As earlier, the solutions are the Bessel functions of the first kind and the corresponding dispersion equation reads

$\eta^{2}=\frac{\varepsilon_{l}^{2}}{r_{0}^{2}}$.

In the cold ion limit, it reproduces Eq. (24). The solutions of Eq. (29) describe the eigen-values of the global eigen-modes in the given cylindrical plasma. Equation (29) can be easily solved numerically for various harmonics by choosing the appropriate $\varepsilon_{l}$ and $m$. In the case of solar coronal magnetic structures, the density and the magnetic field have higher values compared to the previous case (Goedbloed \& Poedts 2004): $n_{\mathrm{i} 0}=n_{\mathrm{e} 0}=$ $10^{16} \mathrm{~m}^{-3}$ and $B_{0} \simeq 10^{-2} \mathrm{~T}$, and the condition (15) now can be rewritten as $2 \pi /\left(m a \rho_{s} \lambda_{z}\right)<1$. Here, we have set $\kappa_{0} \simeq a r_{0}$. Taking as an example a magnetic column with the diameter of $200 \mathrm{~km}$ and, in the case when the density at its edge is 0.1 of its value at the column axis, we get $a \simeq 7 \times 10^{-10} \mathrm{~m}^{-2}$, and therefore, the poloidal mode number $m$ takes very high values $\sim 10^{5}$.

\section{Conclusions}

As seen from Figs. 1, 2, and 4, the frequency of the Alfvén modes depends on the density gradient, and on the coupling with the corresponding drift mode which is driven by the density gradient. The drift mode is practically always unstable and its effects are hardly avoidable in any realistic case. Therefore, the change in the frequency of the Alfvén modes should be taken into account in the analysis of observed modes in the solar corona. In fact, this introduces a certain freedom in the fitting of observations into the theoretical modeling. We note in particular that the widely used one-fluid (MHD) model is intrinsically unable to describe these phenomena. Another consequence of the analysis is that the Alfvén modes are generally damped, and this remains true even if they are coupled to an unstable mode like the drift mode studied here, and even in an environment like the solar corona which is generally assumed as collision-less. Clearly, the coupling of the unstable drift mode to the damped Alfvén mode has some potential for the coronal heating problem. As a matter of fact, the unstable drift modes will always be present in the solar corona and, in turn, excite the Alfvén modes, which are damped and thus contribute to the heating and the acceleration of the coronal plasma. Of course, this is only a qualitative picture and a more detailed modeling and study is required to get a more quantitative idea of the efficiency of this mechanism.

According to the typical parameter values, the electron mean free path $v_{T \mathrm{e}} / v_{\mathrm{e}}$ is comparable to the wave-length in the parallel direction so that the kinetic instability of the drift wave for the given parameters may become important and should be taken into account. Consequently, the present two-fluid description, although introducing some novel phenomena that are as a rule not taken into account in the modeling of solar plasma, omits some physical effects that may become essential. In fact, this problem holds for any fluid modeling, including MHD. The present study should be understood only as a first step in a better description of the Alfvén modes that are coupled to other plasma modes which, on the other hand, are overlooked in the standard MHD approach.

Recently, the detection of relatively high frequency modes $(\sim 10 \mathrm{~Hz})$ in the solar atmosphere has become possible (DeForest 2004). The drift frequencies discussed here are within this limit and these frequencies can be easily made much lower for a larger density scale length $L_{N}$ [bearing in mind the condition (15)]. The same holds for the Alfvén frequencies which can be made small by simply taking longer parallel wave lengths, as it is usually done in the literature. Yet, in our view, for such long scales the validity of the model becomes questionable as vertical stratification and gravity effects should be taken into account then.

Acknowledgements. The results presented here were obtained in the framework of the projects G.0451.05 (FWO-Vlaanderen), C90203 (Prodex), and GOA/2004/01 and OT/02/57 (K.U. Leuven). Authors are grateful to Prof. J. Weiland for invaluable comments and suggestions.

\section{References}

DeForest, C. E. 2004, ApJ, 617, L89

Giaretta, D. L. 1979, ApJ, 75, 237

Goedbloed, J. P., \& Poedts, S. 2004, Principles of Magnetohydrodynamics (Cambridge: Cambridge Univ. Press)

Goldston, R. J., \& Rutherford, P. H. 1995, Introduction to Plasma Physics (Bristol: Institute of Physics Pub.), 376

Krall, N. A., \& Trivelpiece, A. W. 1973, Principles of Plasma Physics (Tokyo: McGraw-Hill Kogakusha), 212

Vernazza, J. E., Avrett, E. H., \& Loeser, R. 1981, ApJS, 45, 635

Vranjes, J., \& Poedts, S. 2004a, Phys. Plasmas, 11, 891

Vranjes, J., \& Poedts, S. 2004b, Phys. Plasmas, 11, 2178

Vranjes, J., \& Poedts, S. 2006a, Phys. Lett. A, 348, 346

Vranjes, J., \& Poedts, S. 2006b, Phys. Plasmas, 13, 032107

Vranjes, J., Petrovic, D., Kono, M., Poedts, S., \& Cadez, V. 2006, Planet. Space Sci., 54, 641

Watson, G. N. 1962, A tretise on the Theory of Bessel Functions (Cambridge:

Cambridge University Press), 482

Weiland, J. 2000, Collective Modes in Inhomogeneous Plasmas (Bristol: Institute of Physics Pub.) 